\title{
A Circuit Approach for Implementing Quantum Memory
}

\author{
Han-Wei Wang, I-Ming Tsai, and Sy-Yen Kuo \\ Department of Electric Engineering, National Taiwan University, Taipei, Taiwan
}

\begin{abstract}
Decoherence is the process that a quantum particle loses its integrity by interacting with the surrounding environment. The problem of decoherence restricts the time available for implementing a given quantum computation process. This phenomenon has a major impact on the performance of quantum computers. In this paper, we propose a scheme to solve this problem. We use a circuit approach to protect our quantum bits (qubit) from decoherence. This results in a quantum memory that has many applications in quantum information science.
\end{abstract}

Index Terms - qubit, coherence time, quantum state, superposition, control-not gate, eigenstate.

\section{INTRODUCTION}

There are many fast and powerful quantum algorithms have been proposed to solve classical time-consuming problems. For examples, Deutsch's algorithm [1] can identify a function as balanced or constant in a single query, Grover's algorithm [2] reduces the time complexity of unordered database search to $\mathrm{O}(\sqrt{N})$, and Shor's algorithm [3] solves the factorization problem in polynomial time. Although these algorithms achieve better performance than their classical counterparts, physical implementation of large-scale quantum computers is still a daunting challenge due to the problem of decoherence.

Driven by quantum fluctuations, a qubit may randomly emit or absorb a photon and lose its coherence [4]. This rapidly destructs the superposition so the system is not able to carry out the correct quantum operation and measurement. This and so our information is lost and we have to start again if this happens.

In a quantum computation system, a qubit can be used to carry quantum information for a period of time during which the quantum state of this qubit is not changed. All operations have to be finished in this duration. This is called the coherence time of this qubit. Although scientists can now create and maintain quantum particles in superpositions for a significant period of time, how to maintain the coherence is still a problem in realizing large scale quantum computing systems.

\section{METHODS OF AVOIDING DECOHERENCE}

There are two main categories on coherence control mechanisms, according to the form of interaction with the system. One of these mechanisms is called open loop technique, the controller acts on the system without obtaining any information about its state. The other is a closed loop technique, the controller acts on the basis of quantum states that obtained by the system. When implementing this quantum device, we have to know the quantum state of the qubit we want to keep. However, this technique is seriously limited by non-ideal detection. Because we can't get the quantum state exactly if the quantum state we measure is not in the eigenstate $\mid 0>$ or $\mid 1>$. Because a measurement on $\alpha|0>+\beta| 1>$ won't get $\alpha|0>+\beta| 1>$, but its eigenstate $\mid 0>$ or $\mid 1>$.

Open-loop techniques are able to decouple an open quantum system from time-varying environment. There are some popular open-loop methods, such as quantum error correction codes and error-avoiding codes. In quantum error correction codes [5]-[7], the errors are mapped onto orthogonal subspaces so that they can be diagnosed and reversed. On the other hand, error-avoiding codes [8]-[12] are based on encoding the state into carefully selected decoherence-free subspaces. However, their main drawback is a large amount of extra-space resources required. There is also a new method which can suppress decoherence by using decoupling pulses [13]. Moreover, these methods can be combined into applications, and sometimes they can overcome both decoherence and design problems [14]-[15]. Our proposal, which is based on a circuit approach, is one kind of the open-loop techniques.

\section{HOW TO MAINTAIN THE QUANTUM STATE WITH OUR QUANTUM CIRCUTT}

We assume that the time for a new qubit to maintain its coherence under certain technology is $T$. Thus, if we transfer the quantum state of the original qubit to a new qubit before it loses its coherence, the new qubit can be regarded as the carrier of the correct information. Because the new qubit is newer than the original one, the information can be stored longer by swapping the states of these two qubits, as shown in Fig.1. In other words, we can transfer the information to a new qubit and make the coherence time longer simply by swapping the state between two particles. In this way, the information won't be destroyed and a longer computation process is feasible. 


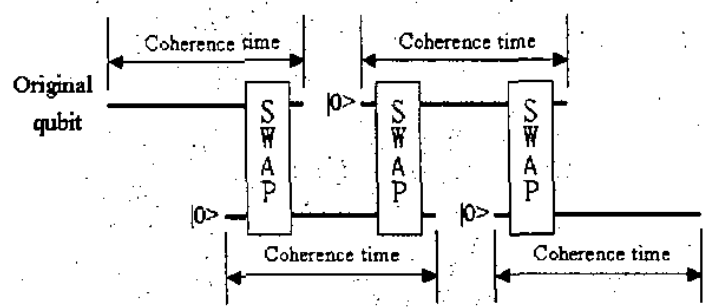

Fig. 1. Maintain the quantum information by swapping the quantum states between the original qubit and the new qubit.

\section{CIRCUIT DESIGN}

\section{A. Quantum state keep circuits for eigenstates}

If the quantum state to be maintained is an eigenstate (i.e. either $\mid 0>$ or $\mid 1>$ ), a single control-not gate can be used to perform the swap operation, as shown in Fig.2. In this circuit, $Q_{0}$ is the initial information carrier and $Q_{t}$ is a temporary information holder. Initially, $Q_{t}$ is set to be $\mid 0>$ before each swap operation. The first control-not gate swaps the quantum information from $Q_{0}$ to $Q_{t}$, a new qubit with longer coherence time.

$$
\begin{aligned}
& \mathrm{CN}\left(\left|0>_{\mathrm{Q}} \otimes\right| 0>\mathrm{T}\right) \\
= & \mathrm{CN}(\mid 00>\mathrm{QT}) \\
= & \mid 00>_{\mathrm{QT}} \\
= & \left|0>_{\mathrm{Q}} \otimes\right| 0>_{\mathrm{T}}
\end{aligned}
$$

or

$$
\begin{aligned}
& \mathrm{CN}(|1>\mathrm{Q} \otimes| 0>\mathrm{T}) \\
= & \mathrm{CN}(\mid 10>\mathrm{QT}) \\
= & \mid 11>\mathrm{QT} \\
= & |1>\mathrm{Q} \otimes| 1>\mathrm{T}
\end{aligned}
$$

After the information is transferred to a new qubit, $Q_{0}$ is reset to its ground state $\mid 0>$ and can be used as the temporary information holder next time. This process can be repeated again to switch the information back to $Q_{0}$, which has a longer coherence time now.

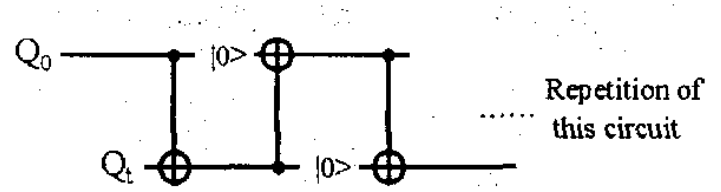

Fig. 2. The quantum state keeper for $\mid 0>$ or $\mid 1>$.

\section{B. Quantum state keep circuits for superpositions}

Similarly to the case shown above, a single qubit in an arbitrary state can be maintained by swapping the information between two qubits. As shown in Fig.3, an auxiliary qubit can be used as the temporary information holder by setting it to $|0\rangle$ first. After the first two controlnot gates, the quantum state of these two qubits are swapped, whatever the quantum state of the initial information holder is.

$$
\begin{aligned}
& \mathrm{CN}\left(\left(\alpha|0>\mathrm{Q}+\beta| 1>_{\mathrm{Q}}\right) \otimes \mid 0>\mathrm{T}\right) \\
= & \mathrm{CN}\left(\alpha\left|00>_{\mathrm{QT}}+\beta\right| 10>\mathrm{QT}\right) \\
= & \alpha|00>\mathrm{QT}+\beta| 11>\mathrm{QT}
\end{aligned}
$$

$$
\begin{aligned}
& \mathrm{CN}(\alpha|00>\mathrm{TQ}+\beta| 11>\mathrm{TQ}) \\
= & \alpha|00>\mathrm{TQ}+\beta| 10>\mathrm{TQ} \\
= & (\alpha|0>\mathrm{T}+\beta| 1>\mathrm{T}) \otimes \mid 0>\mathrm{Q}
\end{aligned}
$$

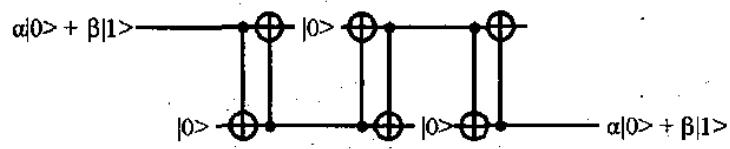

Fig. 3. The circuits for maintaining a single qubit of general quantum state.

\section{ANALYSIS}

The relation between the coherence time and the swap frequency is shown in Fig.4. If $\mathrm{T} 1$ decreases, the frequency of swap operation will increase, then more computation energy or resources will be used. If $\mathrm{T} 1$ increases, the frequency of swap operation decreases. This results in a short $\mathrm{T} 2$, which means the swap operation is closer to the coherence time limit and the information may not be safe. So, there is a trade-off between the resources used in implementing the circuits and the risk of losing the information. Besides, the length of coherence time of each qubit may not be the same under various technologies. We must take this into consideration. As a result, to keep the information for a duration $T$, the lower bound on the number of swap operations to be performed is $\mathrm{T} / \mathrm{T} 1$. 


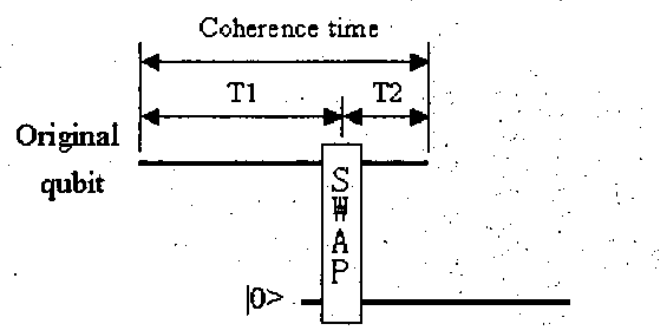

Fig. 4. Analysis on the frequency of swap operations.

\section{APPLICATIONS}

The subject of decoherence is a serious problem of implementing quantum memory. We have to make sure the data can be stored for a long time and won't be influenced by the environment. The mechanism we proposed can be generalized to protect more qubits from decoherence. In addition, we can reuse the temporary qubits to save resources.

As shown in Fig.5., the temporary qubit can be reused in a row of quantum memory cells as a temporary swapping information holder. Notice that the temporary qubit must be set to its ground state before each swapping operations. After applying to the end of the quantum memory, we take the temporary qubit back again as the information holder for the first qubit. Thus, we need only one temporary qubit for one-dimension quantum memory. Notice that the total time of the swap operations must not exceed the coherence time of the first qubit.

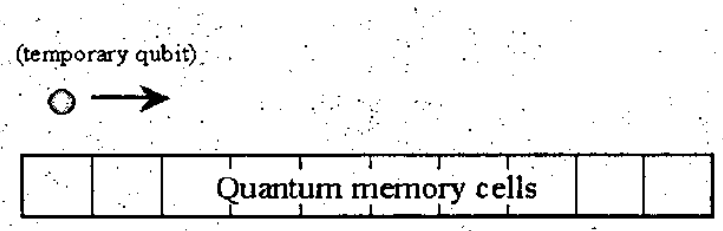

Fig. 5. Temporary qubit reused for one-dimension quantum memory.

\section{CONCLUSION}

How to maintain quantum coherence of a qubit is an important issue in physical realization of quantum computers. There are also many other applications that need to maintain the qubit coherence, for examples, quantum circuit delay, qubit communication channel and quantum error correcting devices. As a result, we know that the requirement and importance of finding the way to solve the coherence problem, when we want to realize quantum devices. Fortunately, we have many choices that can be used to avoid qubit decoherence. We can choose the most applicable ones according to the technology we have and the resources it costs. The circuits we proposed in this paper can be used to solve the decoherence problem with reasonable resources. The only resources required are one temporary qubit and control-not gates. We believed that this is a practical way of implementing quantum memory.

\section{REFERENCES}

[1] David Deutsch, "Quantum Theory, the Church-Turing Principle and the Universal Quantum Computer", Proc. of Royal Society London, A, 400:97-117, 1985.

[2] LK Grover, "Quantum Mechanics Helps in Searching for a Needle in a Haystack", Phys. Rev. Lett.79,p.325, 1997.

[3] P.W. Shor "Algorithms for quantum computation: discrete logarithm and factoring", in S. Goldwasser (Ed.): Proc. 35th A. Symp. on the Foundations of Computer Science, p.124, IEEE, Los Alamitos, CA, 1994.

[4] P. Mohanty and R. A. Webb "Decoherence and quantum fluctuations", Phys. Rev B.55, R13452, 1997.

[5] P. W. Shor "Scheme for Reducing Decoherence in Quantum Computer Memory", Phys. Rev. A 52, 2493, 1995.

[6] A. M. Steane "Error Correcting Codes in Quantum Theory", Phys. Rev. Lett. 77, 793, 1996.

[7] E. Knill and R. Laflamme "Theory of Quantum ErrorCorrecting Codes", Phys. Rev. A 55, 900, 1997

[8] D.A.L.,I.L.Chuang \& K.B.Whaley "Decoherence-Free Subspaces for Quantum Computation", Phys. Rev. Lett. 81, $2594,1998$.

[9] D.A..L. and L.-A. Wu "Reducing Constraints on Quantum Computer Design by Encoded Selective Recoupling", Phys. Rev. Lett. 88, $017905,2002$.

[10] P. Tombesi, D. Vitali "Physical realization of an environment with squeezed quantum fluctuations via QNDmediated feedback"; Phys. Rev. A 50, 4253, 1994.

[11] D. Vitali, P. Tombesi, and G.J. Milburn "Quantum-state protection in cavities", Phys. Rev. A 57, 4930-4944, 1998.

[12] D. Vitali and P. Tombesi "Using parity kicks for decoherence control”, Phys. Rev. A 59, 4178-4186, 1999.

[13] L.-A. Wu and D.A.L. "Creating Decoherence-Free Subspaces Using Strong and Fast Pulses", Phys. Rev. Lett. $88,207902,2002$.

[14] D.A.L.,D.Bacon and K.B. Whaley "Concatenating Decoherence-Free Subspaces and Quantum Error Correcting Codes", Phys. Rev. Lett. 82, 4556, 1999.

[15] M.S. Byrd and D.A.L. "Comprehensive Encoding and Decoupling Solution to Problems of Decoherence and Design in Solid-State Quantum Computing", Phys. Rev. Lett. 89, 047901, 2002. 